\title{
Impact of Decentralised Control in Cerebral Blood Flow Auto-Regulation using 1D and 3D Models
}

The Circle of Willis is a ring-like structure of blood vessels at the base of the brain that distributes oxygen-rich arterial blood to the cerebral mass at a specific rate. Onedimensional (1D) and three-dimensional (3D) Computational Fluid Dynamics (CFD) models of the Circle of Willis have been created to simulate clinical scenarios, such as stenoses and occlusions in afferent arteries. Both models capture cerebral haemodynamic auto-regulation using a Proportional-Integral (PI) controller to modify efferent artery resistances to maintain optimal efferent flow rates for a given circle geometry and afferent blood pressure. Auto-regulatory control is decentralised so that each individual territory of the cerebral mass regulates flow to satisfy its requirements independent of the remaining territories. Results show that if a sudden occlusion is imposed in an afferent artery, efferent flux profiles fluctuate as an equilibrium is found that best satisfies the independent requirements of each territory of the cerebral mass. Clinically, this unique insight into the transient dynamics of cerebral blood flow and auto-regulation will enable pre-surgical scenario testing using simple models to minimise stroke risk.

Keywords: Circle of Willis; Cerebral haemodynamics; Computational Model; Auto-regulation; PI controller 


\section{Introduction}

The Circle of Willis (CoW), shown in Figure 1, is a ring-like structure of blood vessels found beneath the hypothalamus at the base of the brain. Its main function is to distribute oxygen-rich arterial blood to the cerebral mass. Although the brain comprises only approximately $2 \%$ of the total body mass, it demands approximately $20 \%$ of the body's oxygen supply. If brain cells are starved of oxygen for more than a few minutes due to decreased flow and/or perfusion pressure, they become permanently damaged.

\section{[Figure 1]}

Afferent arteries supply the circulus arteries with fresh oxygen-rich arterial blood from the heart. These are the Basilar Artery (BA) and the right and left Internal Carotid Arteries (ICA's). The circulus arteries distribute oxygenated blood from the afferent arteries to the efferent arteries through the CoW. The circle is composed of the Anterior Communicating Artery (ACoA), Left and Right proximal Anterior Cerebral Arteries (LACA $A_{1}$ and $\mathrm{RACA}_{1}$, respectively), Left and Right Posterior Communicating Arteries (LPCoA and RPCoA, respectively), and Left and Right proximal Posterior Cerebral Arteries, $\left(\mathrm{LPCA}_{1}\right.$ and $\mathrm{RPCA} \mathrm{A}_{1}$, respectively). Of the efferent arteries labelled in Figure 1, the major ones are the left and right Anterior Cerebral Artery $\left(\mathrm{ACA}_{2}\right)$, Posterior Cerebral Artery $\left(\mathrm{PCA}_{2}\right)$, and Middle Cerebral Artery (MCA).

The four vascular territories supplied by these six major efferent arteries are the lateral, medial, superior and inferior territories. The lateral, superior and inferior territories are 
irrigated mainly by the MCA's, and the medial territories are irrigated mainly by the ACA's (Ketchum, 2003). Note that each efferent artery predominantly supplies a particular cerebral volume and there is no redundancy in that supply, however there is very minor collateral supply across the brain at the level of the arterioles.

Irrespective of pressure variations in the afferent vessels, the circulus vessels comprising the CoW allow for a constant supply of blood to be delivered to the cerebral mass via the efferent vessels. This supply is regulated by vasoconstriction and vasodilation of the small arterioles in the vascular bed at the end of the efferent arteries. Hence, blood supply, which must be maintained in a tight range, is controlled by changes in the variable downstream resistance.

Ideal distribution of blood depends largely on the anatomical structure of the CoW being complete, however many different abnormalities have been observed, such as absent, fused, string-like, and accessory vessels (Alpers and Berry, 1963). While an individual possessing one of these variations may under normal circumstances suffer no ill effects, there are certain clinical conditions, which compounded with effects of an absent or altered vessel, can lead to an ischaemic stroke.

Clinically, modelling can enable better clinical decisions, by identifying at-risk cerebral arterial geometries prior to surgery or other clinical procedures. To be suitable for realtime clinical decisions the model must be computationally fast, for which the 1D model is particularly relevant and effective. Prior research has resulted in a variety of 
computational solutions (Hillen and Drinkenburg, 1988; Cassot et al., 2000; Lodi and Ursino, 1999; Hudetz et al., 1982; Ferrandez et al,. 2002), most of which either neglect auto-regulation, or ignore the clinically important transient dynamics that this research focuses on.

This research examines the decentralised nature of cerebral control to determine its impact on autoregulation and supply during clinical events. Results from a $1 \mathrm{D}$ and a 3D model of the CoW are compared. Both models capture the dynamics of the autoregulation phenomenon using a PI feedback controller to modify arteriole resistances from a reference value to maintain optimal flowrates to the cerebral mass. These variable, state dependent resistors are modelled as porous blocks, per Ferrandez et al. (2002) and capture the locally controlled behaviour of these systems.

\section{Methodology}

\subsection{Geometry}

A three-dimensional arterial $\mathrm{CAD}$ model of the $\mathrm{CoW}$ was developed from a Magnetic Resonance Angiogram (MRA) of an individual's cerebrovasculature, with artery lengths from the MRA data, and artery diameters from Hillen and Drinkenburg (1988). For simplicity, only flow through the major efferent arteries labelled in Figure 1 is considered.

\subsection{D Model Geometry}


Figure 2 shows a schematic representation of the 1D CoW model, including efferent and afferent arteries, and a sign convention for flow. The model uses the Poiseuille flow approximation where the resistance of an arterial segment is inversely proportional to the fourth power of the vessel radius. Afferent and circulus arteries are shown with constant resistances, as it is assumed that constriction and dilation of the artery walls by smooth muscle cells does not occur to a significant degree in large relatively rigid cerebral arteries (Fung, 1990). Efferent arteries are shown with time-varying resistances that represent the combined resistance to flow of the vascular bed.

\section{[Figure 2]}

\subsection{D Model Geometry}

The computational domain for the $3 \mathrm{D}$ model was created by meshing the CAD reconstruction from an MRA scan. The efferent arteries continually bifurcate downstream of the CoW. Due to the computing power required, the major efferent arteries are terminated a short distance downstream of the CoW with a porous block representing the effects of the capillary bed. The resulting solid model is shown in Figure 3.

\section{[Figure 3]}

\subsection{Dynamic Auto-Regulation for 1D and 3D Models}

The autoregulatory response of the vascular bed resistance is modelled by a PI controller where the control input $u(t)$ is defined: 


$$
u(t)=K_{p} e+K_{i} \int e d t
$$

where $e=\left(q_{r e f}-q\right)$ is the error in the flowrate, and $K_{p}$ and $K_{i}$ are proportional and integral feedback control gains. Feedback control is designed to regulate the flow in an efferent artery, $q$, to its optimal or reference value, $q_{\text {ref, }}$, by altering the efferent resistance to flow, a result achieved physiologically by vasodilation and vasoconstriction at the arterioles. The regulatory mechanism acts on changes in mean arterial pressure, and as such pulsatile flow is not represented. Control gains $K_{p}$ and $K_{i}$, are determined using clinical data from Newell and Aaslid (1994) by matching the percentage change and duration of the time-dependent velocity profiles measured using transcranial Doppler (TCD) recordings of the MCA.

The dynamic behaviour of the peripheral resistance, modelled by the porous block in each model, can be described by a simple first order system:

$$
\tau \dot{R}+\left(R-R_{r e f}\right)=u(t)
$$

where $\tau$ is the time constant of the auto-regulatory response, $u(t)$ is a control input defined by Equation (1), $R$ is the actual resistance of the efferent artery, and $R_{r e f}$ is the resistance of the artery for optimal perfusion for any input condition.

Afferent and circulus arteries are modelled with constant resistances (Fung, 1990), whereas efferent vessels exhibit a time-varying resistance capable of variations between 
$5 \%$ and $195 \%$ of the 'normal' resistance (Newell and Aaslid, 1994; Gao et al., 1998; Mancia and Mark, 1983), an approximation based on observed results from the literature.

The complete CoW is modelled with venous pressures of $15 \mathrm{mmHg}$, and an arterial pressure drop from $100 \mathrm{mmHg}$ to $80 \mathrm{mmHg}$ in the RICA is used to determine control gains for the efferent arteries per Newell and Aaslid (1994) and employed by Ferrandez at al. (2002). The $\mathrm{ACA}_{2}$ and $\mathrm{PCA}_{2}$ are assumed to have a similar, approximately 20 second, response profile.

\subsection{D Fluid Model}

In this case, the $\mathrm{CoW}$ is modelled as a 1D structure with laminar, viscous and incompressible flow. Per Ferrandez et al. (2002), the flow in any arterial element is assumed to be Newtonian and axi-symmetric. Therefore, it can be modelled by the Poiseuille Equation for flow in a tube, where the flowrate, $q$, is a function of the pressure gradient along the vessel, $\Delta \mathrm{p}$, and the resistance, $\mathrm{R}$, to blood flow; and the resistance is a function of the artery length, $l$, artery radius, $r$, and dynamic viscosity of blood, $\mu$ :

$$
\begin{gathered}
q=\frac{\Delta p}{R} \\
R=\frac{8 \mu l}{\pi r^{4}}
\end{gathered}
$$

Equation (4) is applied to each arterial element in Figure 2, and combined with equations for the conservation of mass and continuity of pressure at each vessel junction in Figure 2 to form a non-linear matrix equation: 


$$
\underline{A}(\widetilde{x}(t)) \tilde{x}(t)=\widetilde{b}(t)
$$

where $\underline{A}$ is a matrix containing resistances for each arterial element, $\widetilde{x}$ is a state vector containing flowrates through those arteries and nodal pressures at the end of the arteries, and $\widetilde{b}$ is a vector containing arterial and venous boundary pressures. Note that if the afferent pressures which drive the system, change dynamically, a time-varying system is created. In addition, the auto-regulatory control input, $u(t)$, that modifies efferent resistances is a function of the states $x(t)$, as seen in applying Equations (1) - (2) to create Equation (5). Hence, the matrix $\underline{A}(\widetilde{x}(t))$ is highly non-linear. This model is an improvement on previous models since it recognises the non-linearity of the system, and uses inner iterations to ensure equilibrium at each time-step, (Moorhead et al., 2003).

\subsection{D Fluid Model}

Flow through the CoW is unsteady, incompressible and viscous. Assuming laminar flow, the governing equations, expressed in integral form, are the continuity and momentum conservation equations, respectively:

$$
\begin{gathered}
\int_{V} \frac{\partial \rho}{\partial t} d V+\oint \rho \mathbf{u} d \mathbf{A}=0 \\
\int_{V} \frac{\partial \rho \mathbf{u}}{\partial t} d V+\oint \rho \mathbf{u u} \cdot d \mathbf{A}=-\oint p \underline{\mathbf{I}} d \mathbf{A}+\oint \overline{\bar{\sigma}} \cdot d \mathbf{A}+\int_{V} \mathbf{F} d V
\end{gathered}
$$

where $\mathbf{u}$ is the $3 \mathrm{D}$ velocity vector, $\mathbf{I}$ is the identity matrix, $\overline{\bar{\sigma}}$, is the shear stress tensor and F represents a momentum source vector used in the implementation of the autoregulation mechanism. This system is solved using the finite volume method. The peripheral 
resistances of the porous blocks are incorporated into the CFD model by defining them similar to a porous zone, requiring a permeability, $k$. To relate the permeability to the peripheral resistance, $R$, a modified form of Darcy's law is used:

$$
k=\frac{A}{R \mu L}
$$

where $A$ is the cross-sectional area of the porous block and $L$ is its length. Darcy's Law is associated with the volume-averaged properties of a flow, but it can be incorporated into the Navier Stokes equations, producing the Brinkman Equation:

$$
\left(\frac{\mu}{k}\right) \mathbf{u}=-\nabla p+\bar{\mu} \nabla^{2} \mathbf{u}
$$

In simulation, the terms on the right side of Equation (9) are already incorporated into the momentum equation. The term on the left side is incorporated by introducing it as a momentum source vector. Assuming an isotropic permeability of the porous block, the momentum equation is modified with an additional momentum source to represent the porous block.

$$
\int_{V}^{\partial \rho \mathbf{u}} \frac{\partial}{\partial t} d V+\oint \rho \mathbf{u u} \cdot d \mathbf{A}=-\oint p \underline{\mathbf{I}} d \mathbf{A}+\oint \overline{\bar{\sigma}} \cdot d \mathbf{A}-\int_{V}\left(\frac{\mu}{k}\right) \mathbf{u} d V
$$




\subsection{Solution Algorithm}

Figure 4 illustrates the solution algorithms for the 1D and 3D models. Despite using the same equations to model autoregulation, the 1D and 3D algorithms differ in terms of how the equations are implemented. The 1D model uses the flow error for the current time step, whereas the 3D model uses the flow error from the previous time step. If the time steps are suitably small, any errors will be minimized.

The 1D model uses inner iterations to ensure equilibrium at each time step. A flowrate error triggers the auto-regulation response modelled by the controller, causing a change in efferent resistance that results in a modified flowrate requiring a different control input and efferent resistance. This process of obtaining improved resistance values and calculating corresponding flowrates is continued until the resistance and flowrates do not change between iterations, representing convergence and equilibrium in Equation (5), at each time step. With the 3D model this inner iteration is not possible. Even though the solution of the Navier Stokes equations is an iterative procedure, altering the peripheral resistances and thus the momentum source vector at each iteration would lead to divergence.

\section{[Figure 4]}

\subsection{Reference Fluxes and Resistances}

Hillen and Hoogstraten (1986) indicate a nominal influx of $12.5 \mathrm{~cm}^{3} \mathrm{~s}^{-1}$ to the CoW. A peripheral resistance ratio based on Hillen and Hoogstraten (1986) is defined as 
6:3:4:75:75 for the ACA, MCA, PCA, AChA and SCbA respectively. Evaluation of the steady state reference fluxes involves altering the permeability of the porous blocks with afferent pressures of $100 \mathrm{mmHg}$ and efferent pressures of $15 \mathrm{mmHg}$ until a total flux of $12.5 \mathrm{~cm}^{3} \mathrm{~s}^{-1}$ is achieved with the resistance ratio of $6: 3: 4: 75: 75$. However, the $3 \mathrm{D}$ model predicts a greater pressure loss through the CoW than the 1D model, particularly in the geometrically complex communicating arteries, the ACoA and PCoA's. To achieve the same efferent fluxes, the $1 \mathrm{D}$ efferent resistances were altered less than $5 \%$ to maintain the same total flux and peripheral resistance ratio.

\section{Results and Discussion}

In a previous study (Moorhead et al., 2004), both the 1D and 3D models were subjected to a $20 \mathrm{mmHg}$ pressure drop in the RICA to observe the transient response for an ideal configuration.

\subsection{Ideal Configuration}

Figure 5 shows that the efferent flux profile for the ipsilateral side of the CoW is virtually identical between models for the balanced configuration.

\section{[Figure 5]}

The flux distribution throughout the circle is considered in Figure 6. Despite using the same CoW geometry, the 3D model experiences much larger pressure losses through the 
ACoA than the 1D model, which assumes Poiseuille flow, resulting in underestimated 1D resistances. The 1D model allows a greater amount of blood flow to be re-routed through the ACoA, whereas the 3D model draws more afferent blood supply through the ipsilateral ICA. Possible reasons for this discrepancy arise from the Poiseuille flow assumptions which do not consider pressure losses due to geometric complexity. More specifically, it is obvious in the 3D model that the blood vessels of the CoW take complex paths through space. Furthermore, the diameters of a number of these segments comprising the circle are not of constant diameter, as approximated by the $1 \mathrm{D}$ model, especially at junctions. There are also problems with the assumption of a fully developed velocity profile, especially in the ACoA, which is a particularly short vessel where a velocity profile may not fully develop.

The discrepancy in circulus flow is overcome by increasing the resistance of the ACoA 9-fold to produce the same effective resistance as seen in the 3D results, and achieve similar flux results, as also shown in Figure 6. A similar effect is seen in the PCoA's, and can be overcome by increasing the PCoA resistance 4-fold.

\section{[Figure 6]}

\subsection{Occlusion of RICA}

A situation is modelled in which the RICA becomes totally occluded. In both 1D and 3D models, a "blood-stealing effect" is observed in which the flux profiles of the MCA and ACA fluctuate as a balance is found that best satisfies the independent requirements of 
each territory of the cerebral mass supplied by each efferent vessel. This effect is clearly evident in Figure 7, where solid lines represent the 3D model and dashed lines show the $1 \mathrm{D}$ results.

\section{[Figure 7]}

The response varies between models because of the different solution methods employed. In the balanced configuration case, in which a $20 \mathrm{mmHg}$ pressure drop was simulated, the gains of the efferent arteries were set to match the clinical data of Newell and Aaslid (1994). Since the autoregulation control algorithm reacts to the magnitude of the flow error, the largest arteries required the smallest gains and the smallest arteries required the largest gains to achieve the same relative response. Therefore, the MCA had a smaller control gain acting on a larger error to give the same relative response as the larger ACA control gain acting on a smaller error.

In the 1D model, when the initial error detected is in the same ratio as the balanced case described above, an equal response would be expected such that no blood stealing would be observed since both arteries would be satisfied at the same time. In contrast, the error in the MCA in Figure 7 is proportionally bigger than that of the ACA, hence in the 1D model, its demands begin to be satisfied first. At time $7.4 \mathrm{sec}$, the ACA has the proportionally larger error, so its demands start to be met, at the expense of the MCA. 
The 3D model employs a different solution method in which the proportional gain plays a far greater role. It is observed that both the ACA and MCA flows begin to return to their reference values immediately after the occlusion, with the MCA returning at a greater rate due to the proportionally larger MCA error as discussed for the 1D model. At time $6.6 \mathrm{sec}$, the MCA suddenly wins the 'tug-of-war', as a result is found that best satisfies the independent requirements of each cerebral territory. Due to lack of clinical data for verification, it is unknown which model has the more accurate clinical response.

\section{[Figure 8]}

Figure 8 illustrates that the result is highly dependent on flow through the communicating arteries which are likely to be very patient specific due to the impact of their specific geometry. As mentioned earlier, the 1D model does not account for geometric complexity in its Poiseuille flow approximation, and as such allows too much flow through the communicating arteries. This effect was compensated for by increasing the resistance of the ACoA 9-fold, and the PCoA's 4-fold. In Figure 8, the thin line represents the response to occlusion when the resistance of the PCoA is increased by a factor of 2.5 instead of 4.0. This result hi-lights the difference between the two models in terms of solving for flowrates through the communicating arteries, and the need for more clinical verification. 


\subsection{Tighter resistance limits on efferent vessels}

Previous results have assumed that the time-varying efferent resistances are capable of variations of $\pm 95 \%$, corresponding to a change in arterial radius of $\pm 40 \%$, (Newell and Aaslid, 1994; Gao et al., 1998; Mancia and Mark, 1983). It is currently thought that these limits are too relaxed, and in particular that they allow the arteries to dilate more than is physiologically observed. Guyton and Hall (2000) present a curve in which blood flow is expressed as a function of arterial pressure. This curve, shown in Figure 9, shows that auto-regulation is efficient between mean arterial pressures of $70 \mathrm{mmHg}$ and $170 \mathrm{mmHg}$, but is ineffective at pressures outside this range, indicating a limit of the autoregulatory system.

\section{[Figure 9]}

A similar curve for a balanced $\mathrm{CoW}$ with resistance limits of $\pm 95 \%$ is shown in Figure 10, which plots the normalised MCA flowrates at different mean arterial pressures. Dashed lines represent the limits of the physiological range usually observed in humans. Of particular note from Figure 10, is that flow is maintained at much lower mean arterial pressures than observed physiologically. More specifically, the model allows efferent vessels to dilate to a greater extent than clinically observed.

\section{[Figure 10]}


The resistance limits were then reduced, such that the allowable resistance value was in the range $0.55 \mathrm{Rref}<\mathrm{R}<1.7 \mathrm{Rref}$. A $7 \%$ dead-band was also employed such that if the error obtained from the solution algorithm was within $7 \%$ of the reference value, the controller was switched off. The resulting curve is shown in Figure 11, and matched the clinical expectation well.

\section{[Figure 11]}

\section{4 "Blood stealing" with tighter resistance limits}

As seen from Figures 10 and 11, the upper resistance limit has negligible effect on blood stealing because at high pressures there is oversupply. However, the lower resistance limit corresponding to dilation greatly affects the blood stealing phenomenon, as seen in Figure 12. More specifically, the tighter the lower resistance limit, the lesser the degree of blood stealing, and the more uniform the response.

\section{[Figure 12]}

Figure 13 shows the 1D and 3D model responses to an occluded ICA, with the resistance limits 0.55 Rref $<R<1.7$ Ref. Note that the $1 \mathrm{D}$ model is shown with both a 4 -fold increase in PCoA resistance and a 2.5-fold increase in PCoA resistance.

[Figure 13] 
An increased PCoA resistance of 2.5 appears to more accurately match results from the 3D model. Both models show a much reduced "blood stealing" response than was the case with resistance limits of $0.05 \mathrm{Rref}<\mathrm{R}<1.95 \mathrm{Rref}$. Although in both resistance limits cases, MCA and ACA flowrates were unable to be restored to the reference values, with tighter limits on dilation, these flowrates did not return as close to reference as in the weaker limits case, as seen in Table 1.

\section{[Table 1]}

In the weak resistance limits case, blood stealing did not occur until flowrates had returned to a particular percentage of their reference value. With tighter limits, this value is never reached, and so blood stealing is greatly minimized.

\section{Conclusions}

1D and 3D CFD models of the Circle of Willis have been created to study auto-regulation of cerebral blood flow for clinical events such as occlusions in afferent arteries, and absent circulus vessels. Autoregulation is modelled by a PI feedback controller, where a deviation from optimal efferent flowrates is feedback controlled by vasodilation and vasoconstriction of the capillary bed. In both models, the RICA was subjected to a 20 $\mathrm{mmHg}$ pressure drop, and the transient flux profiles exhibited excellent correlation for two geometries. However, to match 1D circulus fluxes to the 3D model and correct for non-Poiseuille flow, the resistance of the ACoA was increased by a factor of 9 , and the 
resistance of the PCoA's was increased by a factor of between 2.5 and 4.0. As a result, the $1 \mathrm{D}$ model is a clinically valid tool given its excellent correlation with more accurate 3D models.

Auto-regulatory control is decentralised so that each individual territory of the cerebral mass regulates flow to satisfy its requirements independent of the remaining territories. With weak dilatory resistance limits, results show that if a sudden occlusion is imposed in an afferent artery, a 'tug of war' scenario occurs, such that efferent flux profiles fluctuate as a balance is found that best satisfies the independent requirements of each territory of the cerebral mass. With physiologically realistic, tighter resistance limits matched to data from Guyton and Hall (2000), this blood stealing phenomenon is minimized, but not eliminated. Hence, clinically, the decentralised aspect of cerebral autoregulation could result in ischaemic stroke after sudden pressure drops due to stenoses or occlusions, where a centralised controller would have a different outcome. 


\section{References}

1. Vander, A., Sherman, J., Luciano, D., "Human Physiology - The Mechanisms of Body Function”, McGraw Hill, Eighth Edition, 2001.

2. Ketchum, K. "NeuroRadiology Tutor." University of Wisconsin, Dept. of Radiology. www.radiology.wisc.edu/Med_Students/neuroradiology/NeuroRad/NeuroRad

3. Alpers, B. and Berry, R. (1963). "Circle of Willis in Cerebral Vascular Disorders, The Anatomical Structure." Archives of Neurology 8: 398-402.

4. Hillen, B. and Drinkenburg, A. (1988). "Analysis of Flow and Vascular Resistance in a Model of the Circle of Willis." Journal of Biomechanics 21(10): 807-814.

5. Cassot, F., Zagzoule, M., Marc-Vergnes, J. (2000). "Hemodynamic role of the circle of Willis in stenoses of internal carotid arteries. An analytical solution of a linear model". Journal of Biomechanics 33 (2000) 395-405.

6. Lodi, C. A., Ursino, M. (1999). "Hemodynamic Effect of Cerebral Vasospasm in Humans: A Modeling Study.” Annals of Biomedical Engineering vol. 27: 257-273.

7. Hudetz, A. G., Halsey, J. H. Jnr., Horton, C. H., Conger, K. A., Reneau, D. D. (1982). "Mathematical Simulation of Cerebral Blood Flow in Focal Ischemia." Stroke 13(5): 693-700.

8. Ferrandez, A., David, T., Brown, M. D. (2002). "Numerical models of auto-regulation and blood flow in the cerebral circulation." Computer Methods in Biomechanics and Biomedical Engineering 5(1):7-19.

9. Fung, Y. C. (1990). “Biomechanics: Motion, Flow, Stress and Growth”. SpringerVerlag Inc., New York. 
10. Newell, D. and Aaslid, R. (1994). "Comparison of Flow and Velocity During Dynamic Autoregulation Testing in Humans." Stroke 25: 793-797.

11. Gao, E., Young, W. L., Pile-Spellman, J., Ornstein, E., Ma, Q. (1998). "Mathematical Considerations for Modelling Cerebral Blood Flow Autoregulation to Systemic Arterial Pressure”. American Journal of Physiology, 274:1023:1031.

12. Mancia, G., Mark, A. L. (1983). "Arterial Baroreflexes in Humans, volume 3 of Handbook of Physiology, Section 2". American Physiological Society, New York.

13. Moorhead, K. T., Doran, C. V., David, T., Chase, J. G. (2003). "Lumped Parameter and Feedback Control Models of the Auto-regulatory Response in the Circle of Willis." Conference Proceedings, World Congress on Medical Physics and Biomedical Engineering 2003.

14. Hillen, B. and Hoogstraten, H. (1986). "A Mathematical Model of the Flow in the Circle of Willis." Journal of Biomechanics 19(3): 187-194.

15. Moorhead, K.T., Moore, S.M., Chase, J.G., David, T. Fink, J. (2004). "1D and 3D Models of Auto-Regulated Cerebrovascular Flow." Proc $26^{\text {th }}$ International Conf of IEEE Engineering in Med and Biology Society (EMBS 2004), San Francisco, CA, Sept 1-5.

16. Guyton, A.C., and Hall, J. E. "Textbook of Medical Physiology", Philadelphia: Saunders, $10^{\text {th }}$ Edition 2000. 


\section{List of Tables and Figures}

Table 1: Percentage of reference resistance achieved after RICA occlusion

Figure 1: Circle of Willis (Vander, 2001)

Figure 2: 1D model Schematic Circle of Willis Representation

Figure 3: Solid Model of circle of Willis Used in Simulations

Figure 4: Solution Flow Diagram

Figure 5: Ipsilateral Response to $20 \mathrm{mmHg}$ Pressure Drop in RICA

Figure 6: Circulus Flux Comparison after 20mmHg RICA Pressure Drop

Figure 7: Ipsilateral Response of MCA and ACA to an occlusion of the RICA

Figure 8: MCA and ACA response to occluded RICA, with smaller PCoA resistance

Figure 9: Effect of increasing arterial pressure on blood flow (Guyton and Hall, 2000)

Figure 10: MCA flowrate as a function of mean arterial pressure

Figure 11: MCA flowrate as a function of mean arterial pressure with tighter resistance limits and a $7 \%$ dead-band

Figure 12: RMCA flowrate in response to occluded RICA - varying resistance limits

Figure 13: Ipsilateral Response of MCA and ACA to an occlusion of the RICA - tighter resistance limits 
Table 1: Percentage of reference resistance achieved after RICA occlusion

\begin{tabular}{|c|c|c|c|c|c|c|}
\hline & \multicolumn{2}{|c|}{ 3D Model } & \multicolumn{3}{c|}{ 1D Model RPCoA x 4 } & 1D Model RPCoA $\times 2.5$ \\
\cline { 2 - 7 } & MCA & ACA & MCA & ACA & MCA & ACA \\
\hline $\begin{array}{c}\text { Tight Limits } \\
(0.55 \text { Rref }<\text { R }<1.7 \text { Rref })\end{array}$ & 52 & 48 & 34 & 34 & 43 & 44 \\
\hline $\begin{array}{c}\text { Weak Limits } \\
(0.05 \text { Rref }<\text { R }<1.95 \text { Rref })\end{array}$ & 71 & 36 & 47 & 65 & 50 & 65 \\
\hline
\end{tabular}


Figure 1: Circle of Willis (Vander, 2001)

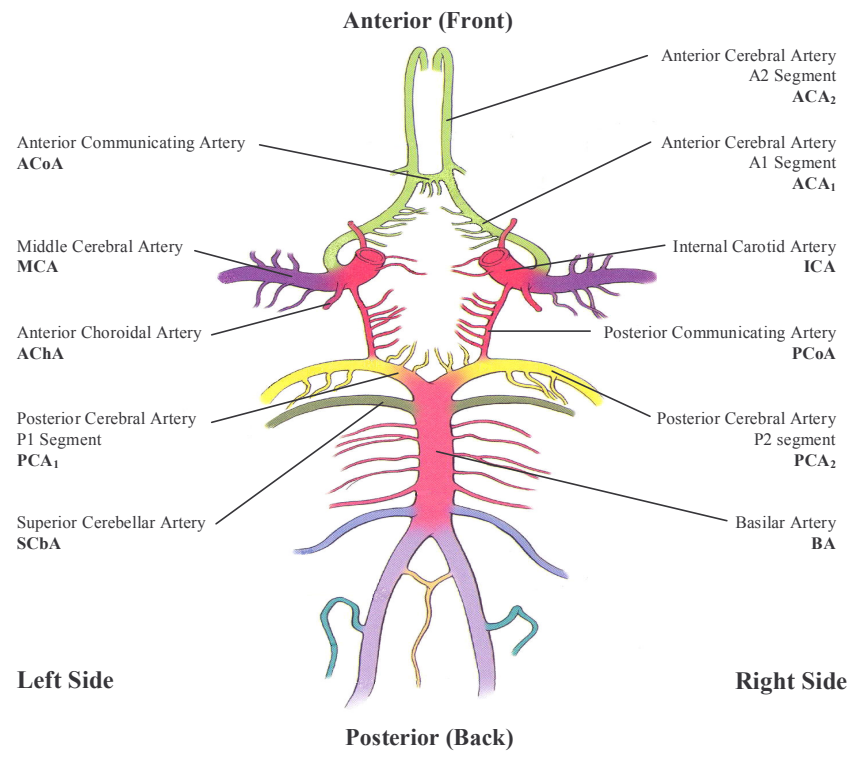


Figure 2: 1D model Schematic Circle of Willis Representation

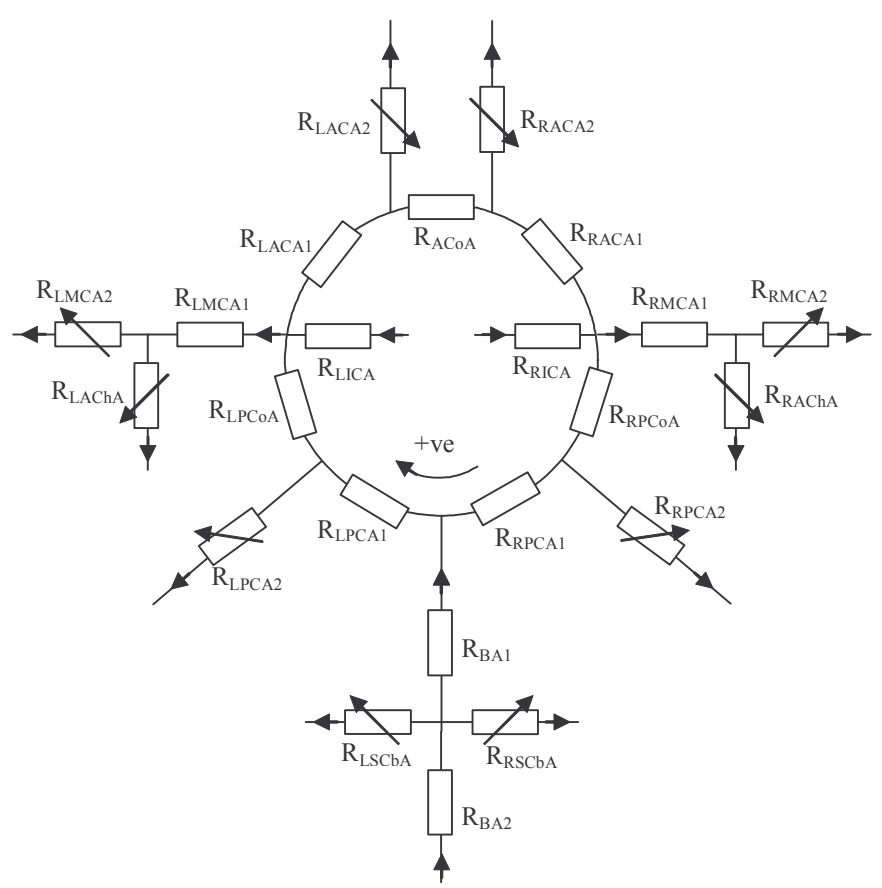


Figure 3: Solid Model of circle of Willis Used in Simulations

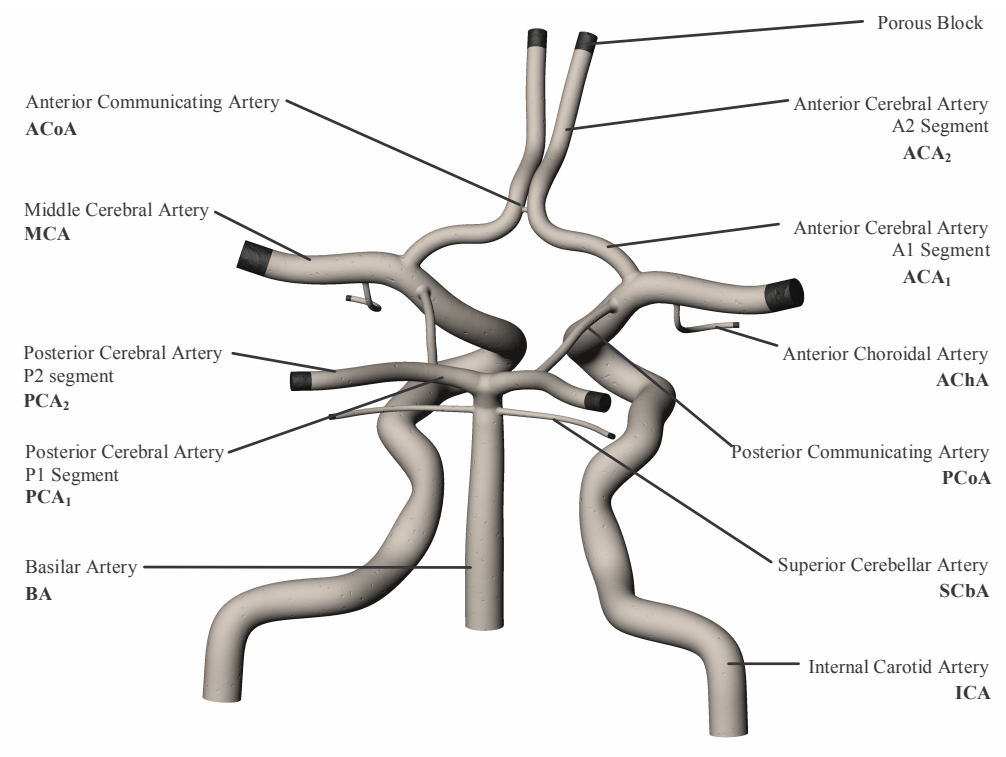

Left Side

Right Side 
Figure 4: Solution Flow Diagram

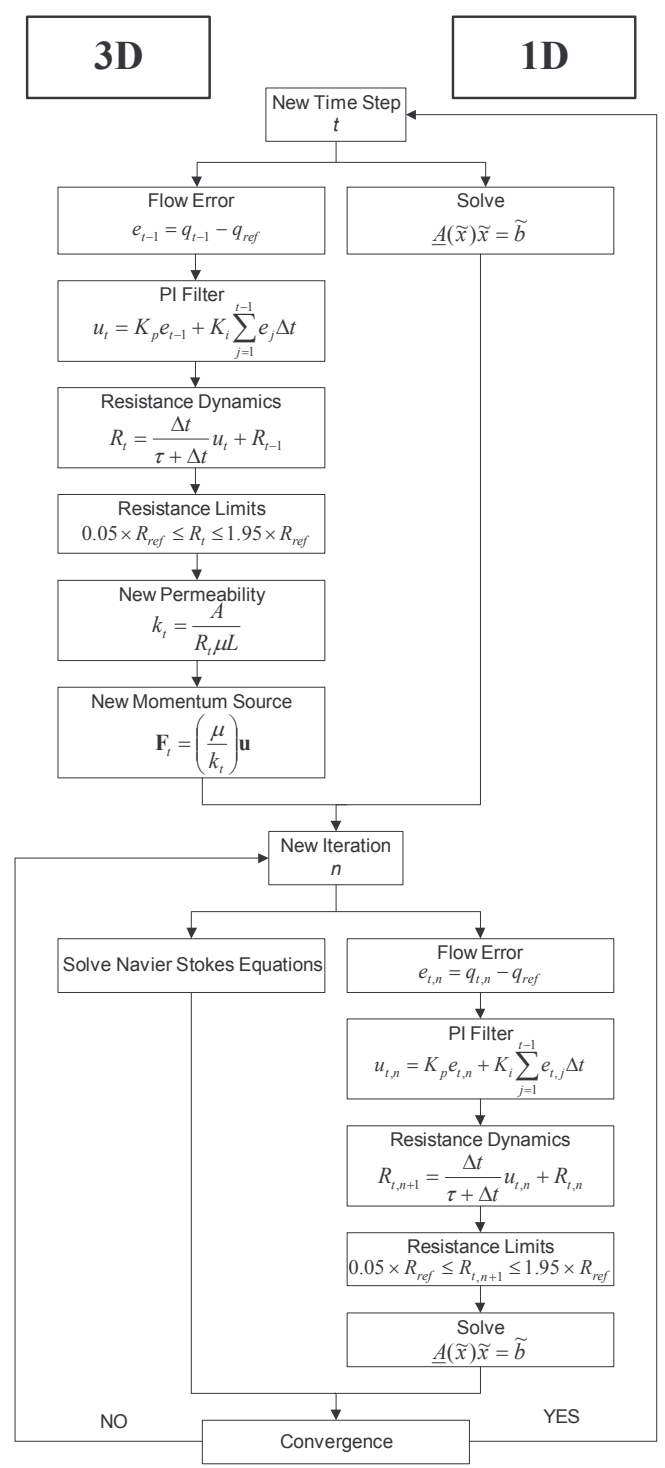


Figure 5: Ipsilateral Response to 20mmHg Pressure Drop in RICA

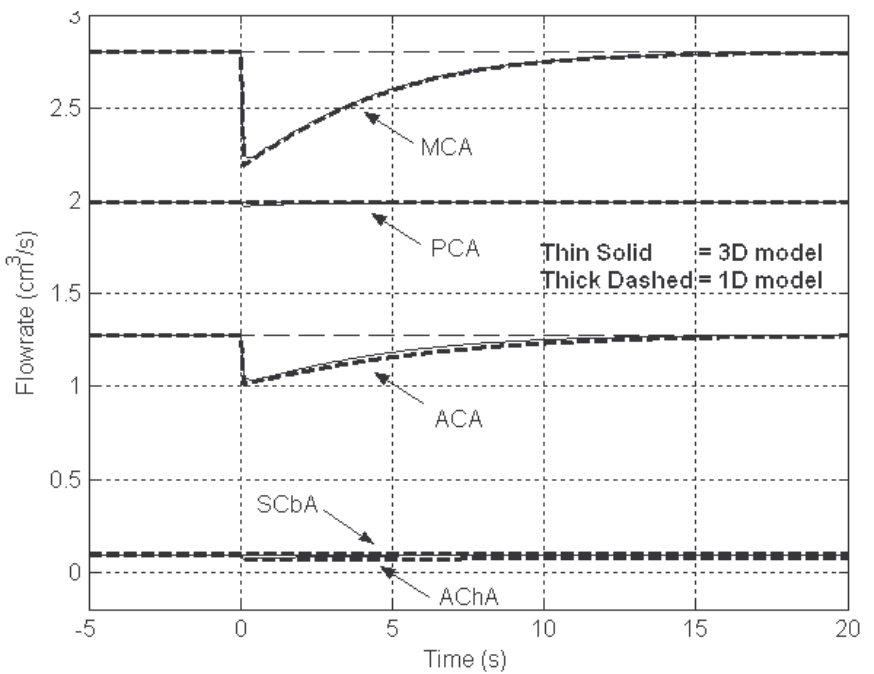


Figure 6: Circulus Flux Comparison after 20mmHg RICA Pressure Drop

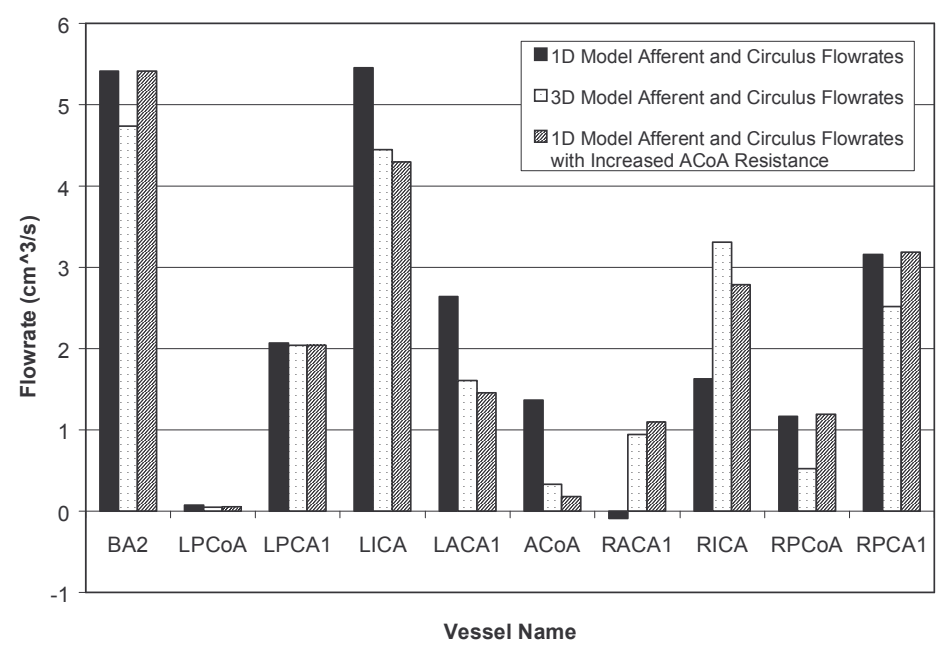


Figure 7: Ipsilateral Response of MCA and ACA to an occlusion of the RICA

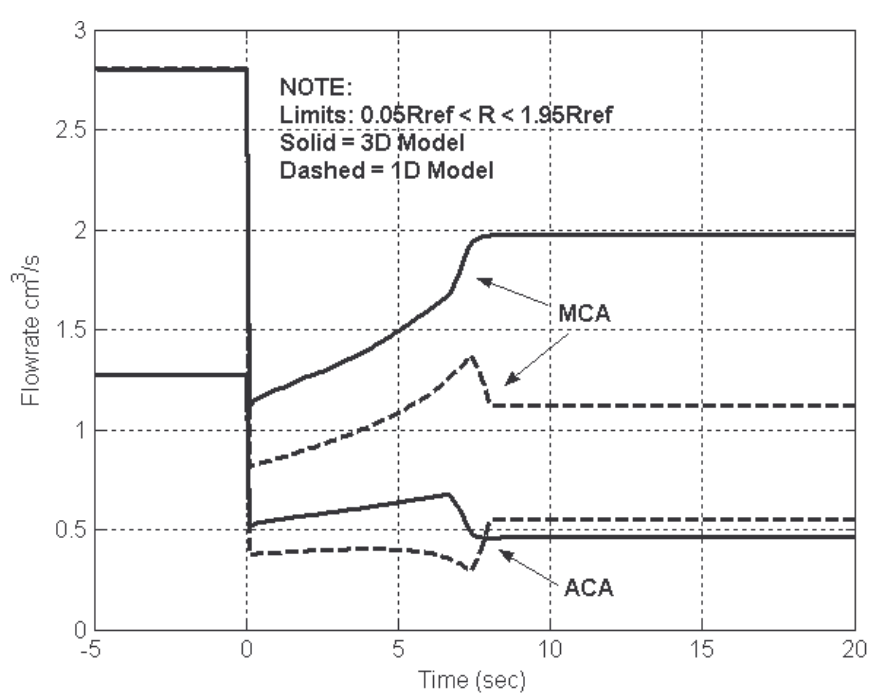


Figure 8: MCA and ACA response to occluded RICA, with smaller PCoA resistance

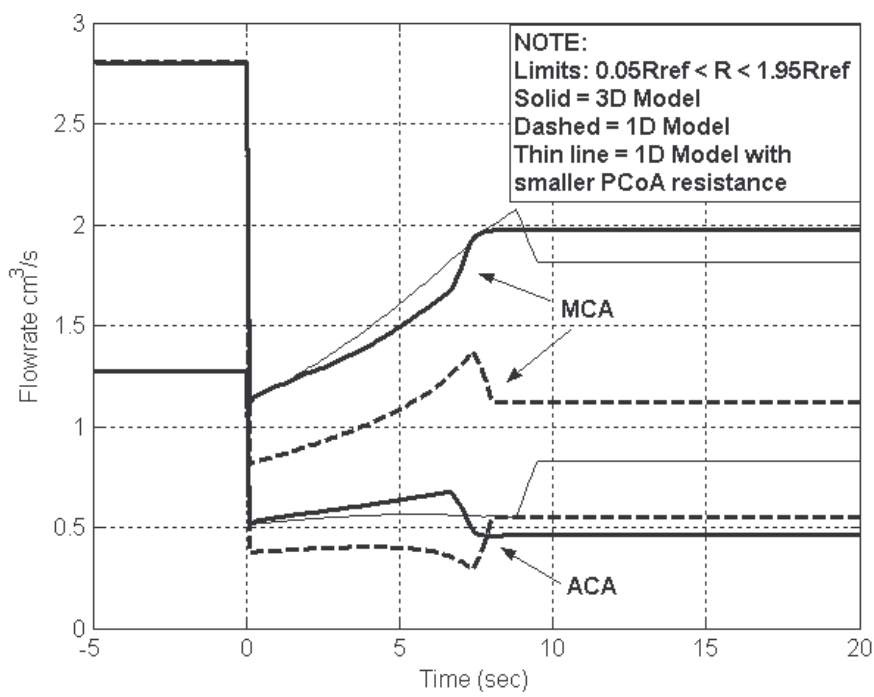


Figure 9: Effect of increasing arterial pressure on blood flow (Guyton and Hall, 2000)

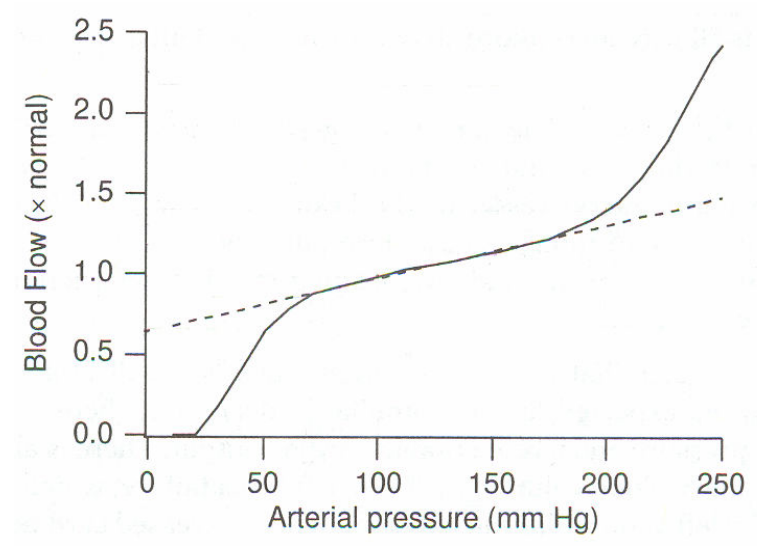


Figure 10: MCA flowrate as a function of mean arterial pressure

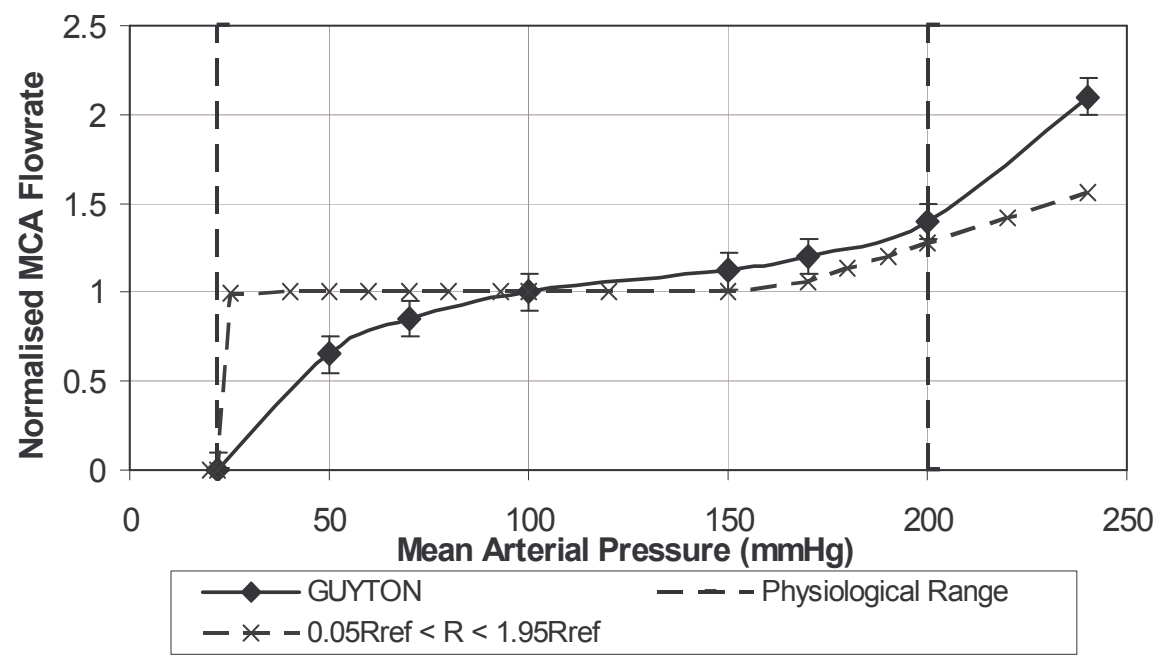


Figure 11: MCA flowrate as a function of mean arterial pressure with tighter resistance limits and a $7 \%$ dead-band

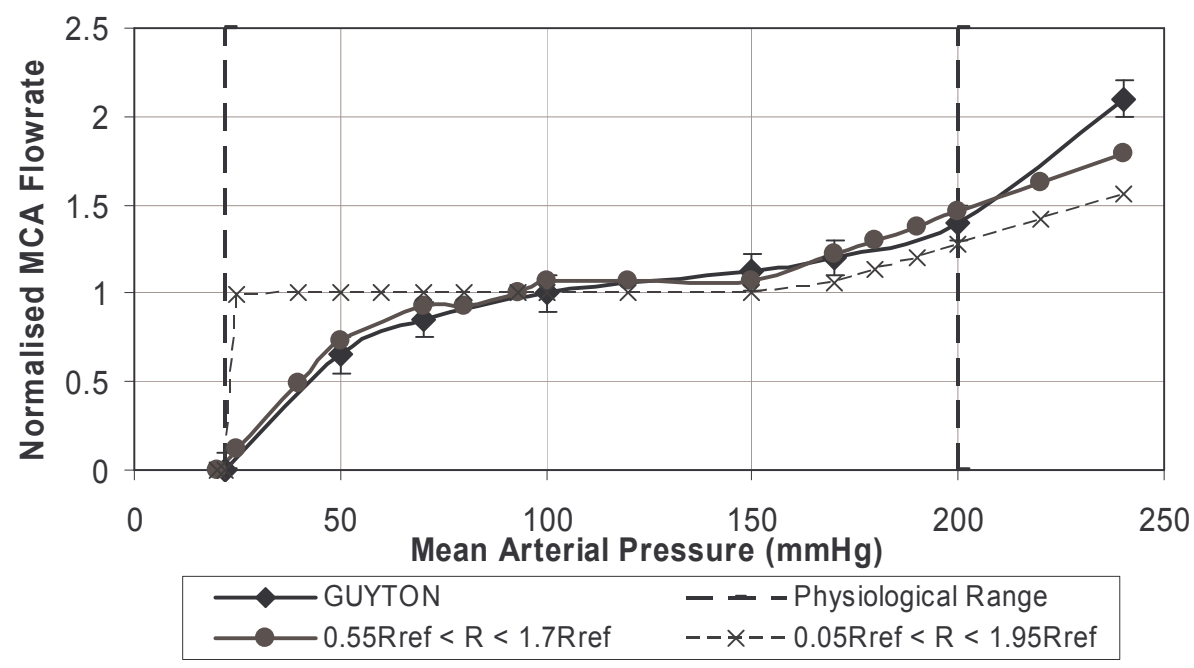


Figure 12: RMCA flowrate in response to occluded RICA - varying resistance limits

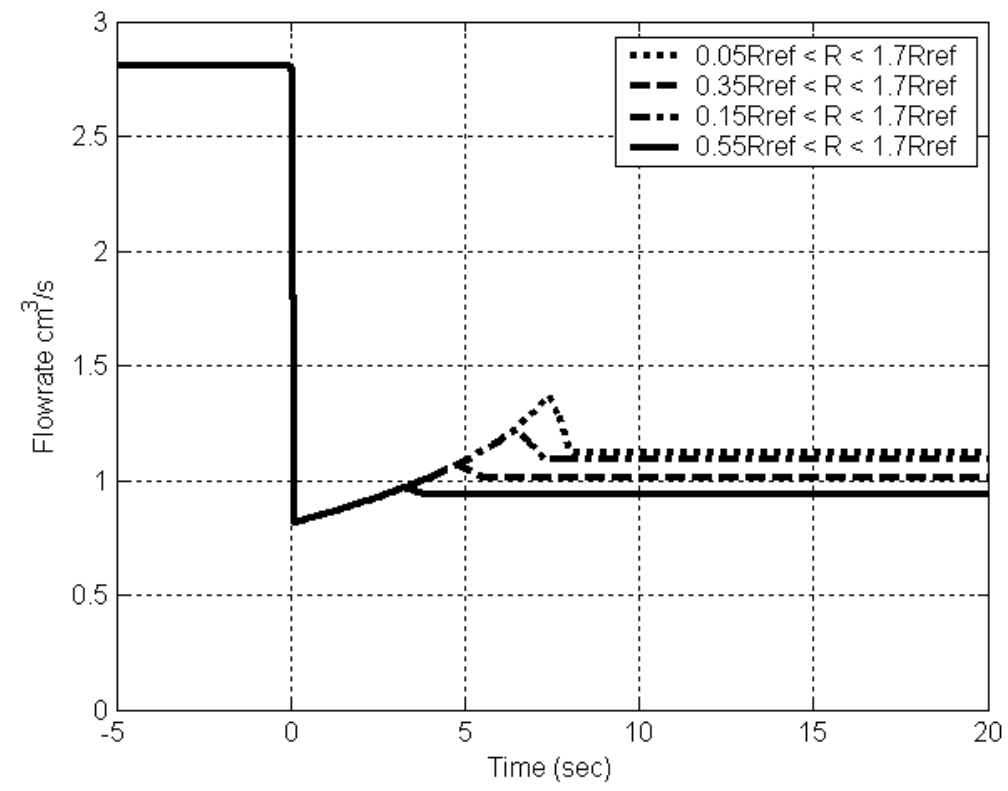


Figure 13: Ipsilateral Response of MCA and ACA to an occlusion of the RICA - tighter resistance limits

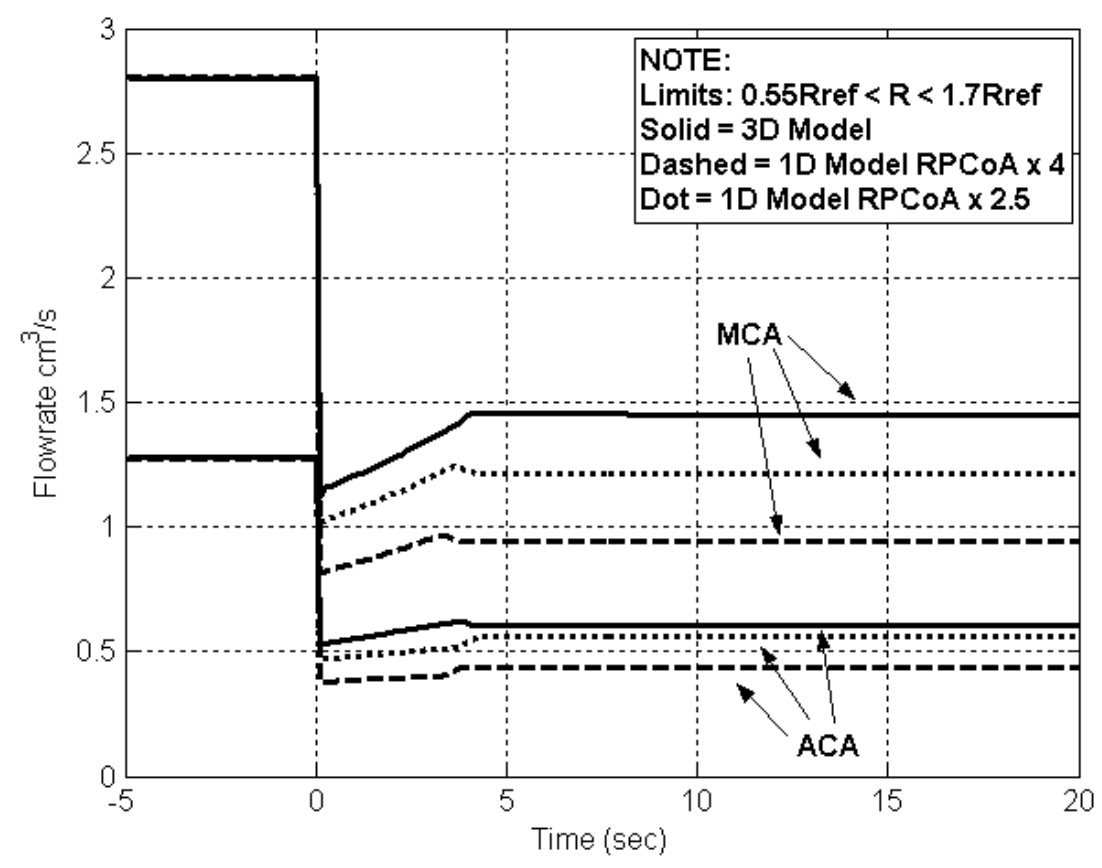

